\title{
Distribución y estructura poblacional del cangrejo Halicarcinus planatus (Brachyura, Hymenosomatidae) en el Canal Beagle, Tierra del Fuego
}

\author{
Distribution and population structure of the crab Halicarcinus planatus \\ (Brachyura, Hymenosomatidae) in the Beagle Channel, Tierra del Fuego
}

\section{Mariano J. Diez ${ }^{1}$, Olga Florentín ${ }^{1}$ y Gustavo A. Lovrich ${ }^{1}$}

${ }^{1}$ Centro Austral de Investigaciones Científicas (CADIC), Consejo Nacional de Investigaciones Científicas y Técnicas (CONICET), Houssay 200 (V9410CAB), Ushuaia, Tierra del Fuego, Argentina. marianodiez@cadic-conicet.gob.ar, marianodiez@hotmail.com

\begin{abstract}
The crab Halicarcinus planatus is the only hymenosomatid that inhabits the southern tip of South America and has a sub-Antarctic distribution, including the peri-Antarctic islands and New Zealand. In this paper, we report the abundance and population structure of $\mathrm{H}$. planatus for a year in the intertidal zone of Bahía Brown, Beagle Channel, Tierra del Fuego. Moreover, we show variations in the population structure in other beaches and environments of the channel. In contrast with males, female density showed monthly variations and was higher during austral summer months. High densities of $\mathrm{H}$. planatus could be related with low mortality during the larval stage and oceanographic features of Bahía Brown, probably enhanced by the presence of both culture and mussel beds, substrates where settlement would occur. The populational sex ratio was biased towards females only in summer, while the operational sex ratio was biased towards males during all periods studied, suggesting strong competition among males for mating. In turn, males were present only in the intertidal zone of Bahía Brown and Macrocystis pyrifera but were absent in the other studied intertidal areas. This is perhaps related to the lack of shelter in the subtidal zone of Bahía Brown. Recruitment occurs in two different periods, summer (J anuaryFebruary) and autumn-winter (May-J une), which corresponds with the two larval cohorts of $\mathrm{H}$. planatus in the Beagle Channel.
\end{abstract}

Key words: Abundance, sex ratio, sexual maturity, recruitment, sub-Antarctic

Resumen. - El cangrejo Halicarcinus planatus es el único himenosomátido que habita el extremo austral de Sudamérica, con una distribución subantártica, incluyendo las islas periantárticas y Nueva Zelanda. En este trabajo, se presenta información sobre la abundancia y estructura poblacional de $\mathrm{H}$. planatus en el intermareal de Bahía Brown, Canal Beagle, Tierra del Fuego durante un año. Además, se muestran las variaciones en la estructura poblacional en otras playas y ambientes del canal. A diferencia de los machos, la densidad de hembras mostró fluctuaciones mensuales en el intermareal de Bahía Brown y fue mayor durante los meses de verano. Las altas densidades de $\mathrm{H}$. planatus podrían relacionarse con la baja mortalidad de estadios larvales y las características oceanográficas de la bahía, probablemente aumentado por la presencia de bancos y cuerdas de cultivo de mejillones, sustratos donde ocurriría el asentamiento de H. planatus. La proporción de sexos poblacional estuvo sesgada hacia las hembras solo en verano, mientras que la proporción sexual operativa se sesgó hacia los machos durante casi todo el período estudiado, sugiriendo una fuerte competencia entre machos por el apareamiento. A su vez, los machos solo estuvieron presentes en el intermareal de Bahía Brown y en los grampones de Macrocystis pyrifera pero no se encontraron en el resto de los intermareales estudiados, probablemente relacionado con la ausencia de refugio en el submareal de Bahía Brown. El reclutamiento existiría en dos períodos diferentes, verano (enerofebrero) y otoño-invierno (mayo-junio), que se corresponde con las dos cohortes larvales de $\mathrm{H}$. planatus presentes en el Canal Beagle.

Palabras clave: Abundancia, proporción de sexos, madurez sexual, reclutamiento, subantártica 


\section{INTRODUCCIÓN}

Las especies de la familia Hymenosomatidae se encuentran principalmente en la región Indo-Pacífica, y el endemismo es una tendencia general de su distribución (Chuang \& Ng 1994). Los himenosomátidos habitan una gran variedad de ambientes, tanto dulceacuícolas como salobres y marinos (Chuang \& Ng 1994, Johnston \& Robson 2005). La mayoría de las especies marinas se distribuyen en aguas someras costeras o en el intermareal, y tienden a ser más abundantes en localidades protegidas como bahías, estrechos y ensenadas. Esta característica hace que muchos himenosomátidos marinos habiten en la proximidad de estuarios y estén expuestos a salinidades más bajas en los tiempos de descarga de agua dulce provenientes de tierra (Lucas 1980, Rahayu \& Ng 2004).

Los cangrejos marinos Hymenosomatidae habitan sobre algas coralinas y filamentosas, bosques de macroalgas, en bancos de mejillones, entre valvas vacías o filamentos del biso, entre ascidias, esponjas o briozoos, y bajo rocas, o enterrados en fango o arena (Lucas 1980). Sin embargo, la mayoría de las especies presentan un hábitat específico y pocas se encuentran en más de un sustrato (Chuang \& Ng 1994). Generalmente, las especies marinas de himenosomátidos no son las más abundantes de las comunidades bentónicas (Pérez-Barros et al. 2004, Diez et al. 2009) aunque pueden presentar altas densidades. Halicarcinus planatus (Fabricius, 1775) constituye una excepción dentro de la familia, ya que no se encuentra sobre un único sustrato sino que puede habitar tanto la zona litoral como los grampones de Macrocystis pyrifera y bancos submareales de mejillones (Richer de Forges 1977, Chuang \& Ng 1994).

El conocimiento sobre los crustáceos decápodos que habitan la zona intermareal del Canal Beagle es escaso (e.g., Avalos 2005), sobre todo en cuanto a la dinámica y estructura de las poblaciones. En el canal las poblaciones intermareales deben responder tanto a la inestabilidad de su ambiente como a los cambios físicos estacionales propios de un ambiente costero subantártico (Hernando et al. 2008 ${ }^{1}$ ). Estas respuestas pueden consistir, por ejemplo, en alteraciones estacionales de las distribuciones de frecuencias de tamaño, la proporción de sexos, entre otros parámetros (Johnston \& Robson 2005). Así, los cambios en los parámetros poblacionales pueden afectar el rol ecológico de la especie (e.g., como presa o procesador de materia orgánica) en el espacio y tiempo. Teniendo en cuenta la importancia ecológica de Halicarcinus planatus como potencial especie clave en el intermareal del Canal Beagle, los objetivos de este estudio fueron evaluar la estructura poblacional anual de Halicarcinus planatus en el intermareal rocoso de Bahía Brown y compararla con diferentes localidades del intermareal del Canal Beagle.

\section{Materiales y MÉtodos}

\section{ÁREA DE ESTUDIO}

El estudio fue realizado principalmente en el intermareal de Bahía Brown, ubicado hacia el Este del Canal Beagle (5451’S; 6730O) (Fig. 1). Bahía Brown es una bahía semi-cerrada que presenta una circulación particular de corrientes, en la que el agua permanece dentro de la bahía durante el descenso de la marea y se renueva durante su ascenso (Hernando et al. 20081). Además, es el único lugar del Canal Beagle donde se realizan cultivos de mejillones por cuerdas suspendidas. El intermareal de la bahía está constituido por rodados del tipo guijones, de aproximadamente $25 \mathrm{~cm}$ de diámetro, apoyados sobre otros guijones y guijarros. La pendiente de la playa es de $6^{\circ}$ y las mareas de sicigia se registran durante el día en primavera-verano y durante la noche en otoño-invierno. La temperatura del agua en el piso bajo del intermareal se encuentra entre 5,2 y $9,5^{\circ} \mathrm{C}$.

\section{COLECCIÓN Y PROCESAMIENTO DE LAS MUESTRAS}

Las muestras se tomaron mensualmente desde noviembre 2006 a octubre 2007, durante los días de mareas bajas de sicigia. En cada mes se recolectaron 5 réplicas con cuadrado de $50 \times 50 \mathrm{~cm}\left(0,25 \mathrm{~m}^{2}\right)$, distribuidas al azar en un estrato del intermareal de Bahía Brown, que se ubicó 0,20$0,25 \mathrm{~m}$ por encima del plano de referencia de las mareas. De cada cuadrado se extrajeron los individuos de $H$. planatus que fueron observados a simple vista, así como los guijarros y el sedimento hasta $5 \mathrm{~cm}$ de profundidad. El material fue tamizado a través de una malla de $1 \mathrm{~mm}$ de abertura para retener los individuos pequeños. Todos los ejemplares fueron fijados en formol salino 6\% tamponado con borato de sodio.

${ }^{1}$ Hernando M, E Torres, N San Román \& M Hoffmeyer. 2008. Monitoreo planctónico y ambiental para el desarrollo sustentable del cultivo comercial del mejillón Mytilus edulis chilensis en la zona de Almanza, Canal Beagle, Tierra del Fuego. Informe final subproyecto AB-62, Proyecto PNUD Arg/ 02/018 GEF. 


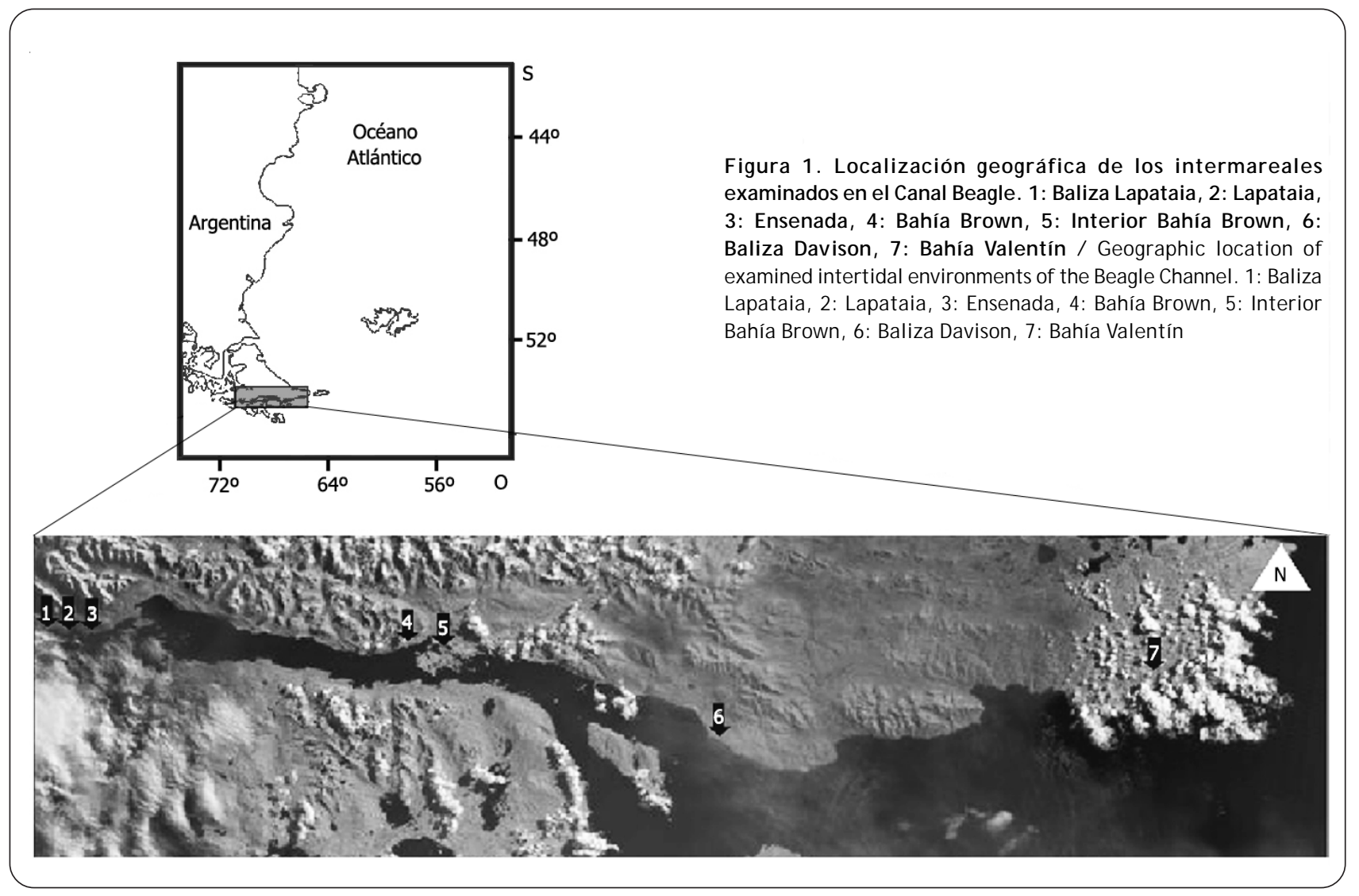

En el laboratorio se registró el total de individuos. Para la determinación del sexo se utilizó la forma del abdomen, de acuerdo a la diferenciación sexual que ocurre en etapas tempranas del desarrollo (Richer de Forges 1977, Vinuesa \& Ferrari 2008a). Las hembras se clasificaron en hembras inmaduras o maduras (Richer de Forges 1977, Vinuesa \& Ferrari 2008a), ovígeras si portaban huevos en sus pleópodos o no ovígeras. El tamaño se estimó a partir del ancho de caparazón (AC), medido a la altura de las dos espinas posteriores sobre una lupa estereoscópica con un ocular grillado. La abundancia se estimó como individuos por $\mathrm{m}^{-2}$ (ind. $\mathrm{m}^{-2}$ ). Con los tamaños se construyeron distribuciones de frecuencias para cada sexo con intervalos de $0,5 \mathrm{~mm}$.

\section{Distribución en el Canal Beagle}

Con el objetivo de evaluar la estructura poblacional de Halicarcinus planatus en otros ambientes, durante octubre 2006 y enero-marzo 2009, se tomaron muestras en diversas playas del Canal Beagle con diferentes características (Tabla 1, Fig. 1). En una de las playas se realizó un muestreo similar al descrito para Bahía Brown.
En las restantes playas, se colectaron individuos de manera aleatoria debido a la dificultad por la fisonomía de las playas de obtener muestras a partir del cuadrante de $0,5 \times 0,5 \mathrm{~m}$. Por otro lado, se utilizaron datos sobre la presencia de $H$. planatus en muestras de grampones de Macrocystis pyrifera colectadas durante los años 1999 y 2000 en la isla Despard (5452’S 68¹0’O) como también una muestra colectada de las líneas de cultivo de mejillones de Bahía Brown de septiembre 2007.

\section{Proporción de sexos}

La proporción de sexos se estimó de dos maneras. Se calculó la proporción de sexos poblacional (PSP) como: $\mathrm{PSP}=\mathrm{n}$ machos $\cdot(\mathrm{n} \text { machos }+\mathrm{n} \text { hembras })^{-1}($ Wilson \& Hardy 2002). Los individuos muestreados durante todos los meses se agruparon para evaluar la distribución de la proporción de sexos en intervalos de tamaño de $0,5 \mathrm{~mm}$. Se calculó la proporción de machos y hembras presentes en cada intervalo. Además, se estimó la proporción de sexos operativa (PSO) es decir, la relación entre machos y hembras listos para aparearse. La relación se estimó como PSO $=$ machos $\mathrm{FM} \cdot(\text { hembras } \mathrm{M}+\text { machos } \mathrm{FM})^{-1}$. Se 


\begin{tabular}{|c|c|c|c|c|}
\hline Sitio & Sustrato & Inclinación & $\begin{array}{l}\text { Oleaje con } \\
\text { viento SO }\end{array}$ & $\begin{array}{c}\text { Macrocystis } \\
\text { pyrifera }\end{array}$ \\
\hline Baliza Lapataia & Arena gruesa con rocas & & Débil & $\mathrm{Si}$ \\
\hline Lapataia & Rocoso & & Débil & $\mathrm{Si}$ \\
\hline Ensenada Oeste & Rocoso & $5^{\circ}$ & Débil & $\mathrm{Si}$ \\
\hline Ensenada Este & Duro con mejillonar & $10^{\circ}$ & Fuerte & $\mathrm{Si}$ \\
\hline Bahía Brown & Rocoso & $6^{\circ}$ & Fuerte & No \\
\hline Interior Bahía Brown & Arena gruesa con mejillonar & $3,5^{\circ}$ & Fuerte & No \\
\hline Baliza Davison & Rocoso & $2,8^{\circ}$ & Mediano & $\mathrm{Si}$ \\
\hline Bahía Valentín & Rocoso & & Fuerte & $\mathrm{Si}$ \\
\hline
\end{tabular}

consideraron machos FM a los de tamaño $\geq$ al tamaño de madurez fisiológica (3,57 mm AC, Diez \& Lovrich 2010). Se consideraron hembras $\mathrm{M}$ a las de tamaños $\geq$ al tamaño de madurez morfométrica (9,55 mm AC, Diez \& Lovrich 2010) ya que es similar al tamaño de madurez fisiológica (Diez \& Lovrich 2010). La PSO es una herramienta que ayuda a explicar los roles de cada sexo y la intensidad en la competencia por aparearse (Kvarnemo \& Ahnesjö 2002). Un sesgo en esta proporción puede predecir cuál de los sexos compite para acceder al apareamiento y describir cómo es la intensidad de esta competencia (Kvarnemo \& Ahnesjö 2002).

\section{AnÁLISIS DE LOS DATOS}

Las variaciones mensuales en la densidad de Halicarcinus planatus obtenidos de Bahía Brown y categorizados todos como, machos, hembras inmaduras, hembras maduras no ovígeras y hembras ovígeras, se compararon mediante ANOVAs de una vía para cada categoría (Sokal \& Rohlf 1995). Los supuestos de normalidad y homogeneidad de varianzas se testearon visualmente mediante la gráfica de los residuos y con el test de Shapiro-Wilks y la prueba de Levene, respectivamente (Sokal \& Rohlf 1995). Si los datos no cumplieron con los supuestos se realizaron análisis de varianza no paramétricos, con la prueba de KruskallWallis. Cuando una prueba ANOVA o Kruskall-Wallis mostró diferencias significativas se realizaron comparaciones múltiples con pruebas a posteriori de Duncan o comparaciones de a pares, respectivamente. Las desviaciones en la proporción de sexos de los valores esperados tanto mensuales como en los intervalos de tamaño, se compararon mediante pruebas $\chi^{2}$ (Sokal \& Rohlf 1995, Wilson \& Hardy 2002).

\section{Resultados}

\section{Abundancia en Bahía Brown}

Entre noviembre de 2006 y octubre de 2007 se colectaron 3329 individuos de Halicarcinus planatus en el intermareal de Bahía Brown: 1837 hembras (55,19\%) y 1492 machos (44,81\%). Se encontraron diferencias significativas en el tamaño promedio $(\mathrm{t}=5,13 ; P<0,001)$ entre machos (5,94 $\pm 2,29 \mathrm{~mm} \mathrm{AC})$ y hembras $(6,34 \pm 2,21$ mm AC), con un rango de tamaño de 1,96-14,52 mm AC para machos y 1,56-12,01 mm AC para hembras. La densidad total de cangrejos mostró variaciones con los meses del año, resultando una densidad promedio mínima de $177,6 \pm 87,9$ y máxima de $302,4 \pm 38,4$ ind. $\mathrm{m}^{-2}$ en junio y enero, respectivamente (Fig. 2). La densidad de cangrejos varió significativamente durante los meses estudiados $\left(\mathrm{F}_{11,59}=2,65 ; P<0,01\right)$ (Fig. 2$)$ : las densidades más altas se observaron durante los meses de verano (enero-febrero) y las más bajas a fines del otoño (junio) y mediados de la primavera (octubre-noviembre) (Fig. 2).

Al discriminar los individuos por sexo se observó que la densidad de Halicarcinus planatus varió mensualmente en las hembras inmaduras $\left(\mathrm{F}_{11,59}=4,94 ; P\right.$ $<0,0001)$ (Fig. 3), hembras maduras no ovígeras $\left(\mathrm{F}_{11,59}=\right.$ 8,67; $P<0,0001$ ) (Fig. 3) y hembras ovígeras $(\mathrm{H}=34,74 ; P$ $<0,001$ ) (Fig. 3) pero no en los machos, que mostraron una densidad promedio mínima y máxima de 78,4 $\pm 26,9$ y $119,2 \pm 19,8$ ind $\mathrm{m}^{-2}$ en noviembre y febrero, respectivamente $\left(\mathrm{F}_{11,59}=1,3 ; P=0,25\right)$. La densidad de hembras inmaduras fue significativamente más alta en verano (enero-marzo) y más baja en invierno (junio-julio; Fig. 3). La densidad de hembras maduras no ovígeras fue similar durante todo el año, excepto en verano cuando fue significativamente más alta (Fig. 3). La densidad de 

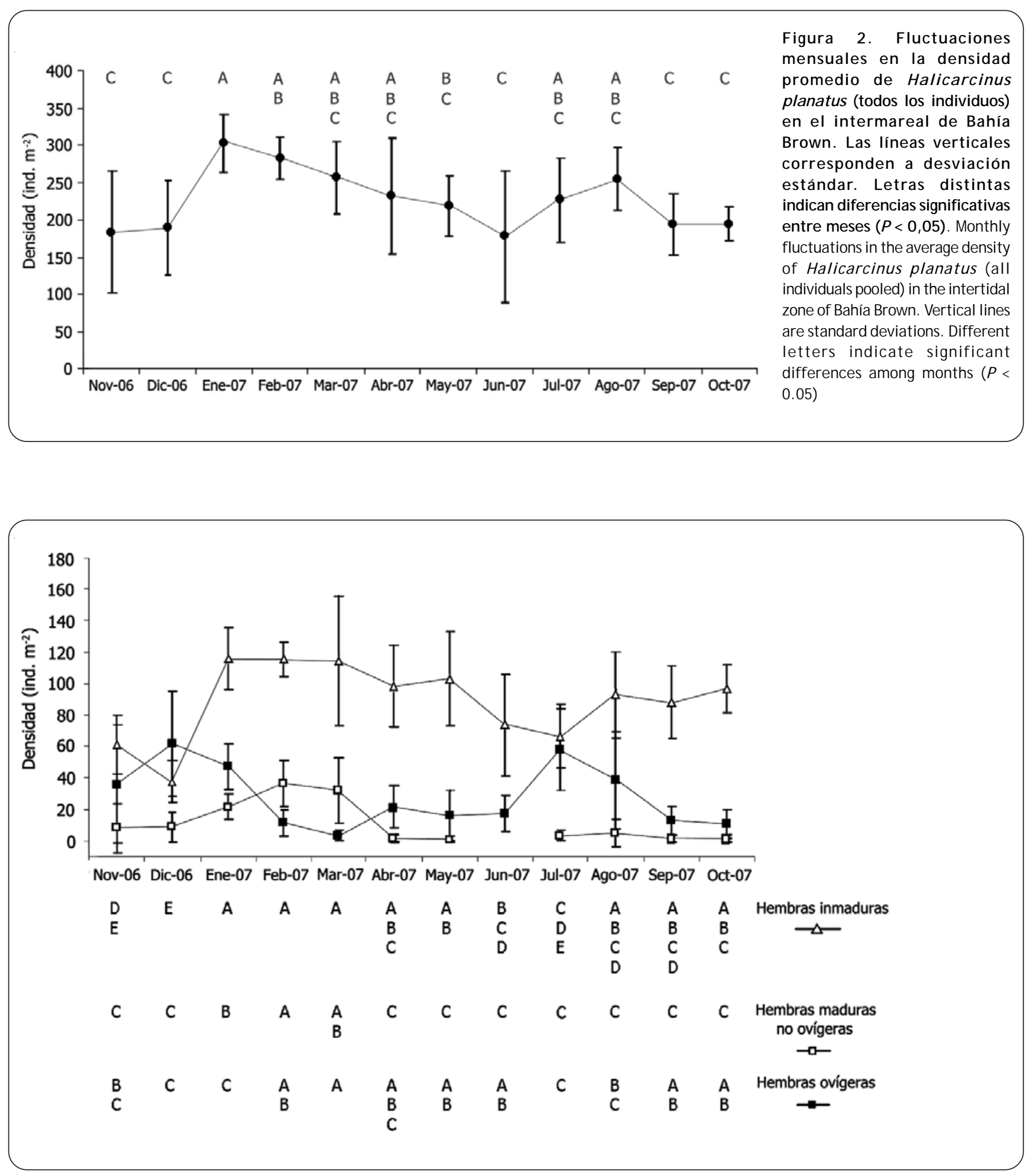

Figura 3. Variaciones mensuales de la densidad promedio de hembras de Halicarcinus planatus clasificadas de acuerdo a su estado de madurez en el intermareal de Bahía Brown. Las líneas verticales corresponden a desviación estándar. Letras distintas indican diferencias significativas entre meses $(\mathbf{P}<\mathbf{0 , 0 5})$ / Monthly variations in the average female density of Halicarcinus planatus classified according to their maturity stage, in the intertidal zone of Bahía Brown. Vertical lines are standard deviations. Different letters indicate significant differences among months $(P<0.05)$ 
hembras ovígeras disminuyó a medida que aumentaba la densidad de hembras maduras, alcanzando un mínimo en verano. Además, las hembras ovígeras mostraron dos picos de densidad máxima, diciembre y julio, ambos coincidentes con disminuciones en la densidad de hembras inmaduras (Fig. 3). La proporción de hembras inmaduras aumentó desde diciembre hasta junio. En diciembre se observó la mínima proporción de hembras inmaduras (37,6\%). En diciembre y julio ocurrieron las máximas proporciones de hembras ovígeras (61,6\% y $57,6 \%$, respectivamente).

\section{Proporción de Sexos en Bahía Brown}

Durante la mayor parte del año la proporción de sexos poblacional de Halicarcinus planatus en el intermareal de Bahía Brown no se desvió de la proporción 0,5 (Fig. 4). Sólo durante los meses de verano la proporción de sexos estuvo sesgada hacia las hembras (Fig. 4). La proporción de sexos operativa mostró variaciones la mayor parte del año. Entre lo meses de febrero y junio, agosto y octubre, se desvió significativamente de la relación 0,5 con predominio de machos (Fig. 4).

Al considerar la proporción de sexos en función del tamaño, se observó que los machos dominaron las clases de tamaño más grandes hasta un $100 \%$, debido a la ausencia de hembras en esos intervalos y de un número bajo de individuos (Fig. 5, Tabla 2). Las clases de tamaño

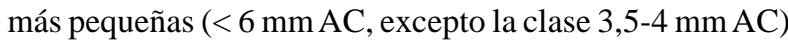
no presentaron desviaciones de la proporción de sexos 0,5 . En contraste, las clases de tamaño intermedias (7,5$10 \mathrm{~mm} \mathrm{AC)} \mathrm{fueron} \mathrm{diferentes} \mathrm{de} \mathrm{la} \mathrm{proporción} \mathrm{0,5} \mathrm{y}$ estuvieron dominadas por hembras (Fig. 5, Tabla 2).

\section{Distribución de FRECuencias de LONGITUd en Bahía Brown}

En general, Halicarcinus planatus presentó una estructura de tamaño diferente para cada sexo. Los machos mostraron una distribución de frecuencia de tamaño sin patrón definido (Fig. 6). Las clases de tamaño dominantes fueron las $<5 \mathrm{~mm}$ siendo más frecuentes en las distribuciones del verano (enero: 62\%, febrero: 53\%, marzo: 62\%) e inicios de la primavera (septiembre: 63\%, octubre: 58\%). Las clases de tamaño superiores (e.g., > $11 \mathrm{~mm}$ ) estuvieron representadas durante todo el período de estudio pero siempre con baja frecuencia (e.g. $\leq 5 \%$ en julio). Particularmente, los tamaños cercanos a 15 mm sólo se encontraron en noviembre (Fig. 6). Las clases de tamaño intermedias 6-11 $\mathrm{mm}$ se observaron durante todos los meses con distintas frecuencias (Fig. 6).

Las hembras de Halicarcinus planatus presentaron distribuciones de frecuencia de tamaño distintas a los machos, visualmente bimodales, con clases mejor representadas que éstos y con altas frecuencias (e.g., mayo: $40 \%$ de los individuos entre 6,5-8,5 mm). Entre noviembre y enero las clases más representadas fueron > 7,5 mm, con las mayores frecuencias para estas clases (73\% de los individuos; Fig. 7). A partir de enero aparecieron los individuos de menor tamaño (3,5-6 mm) con aumentos en su frecuencia en cada mes (Fig. 7). Por otra parte, durante el período estudiado no se encontraron estadios juveniles tempranos (i.e., cangrejo I, 1,1 mm $\mathrm{AC)}$.

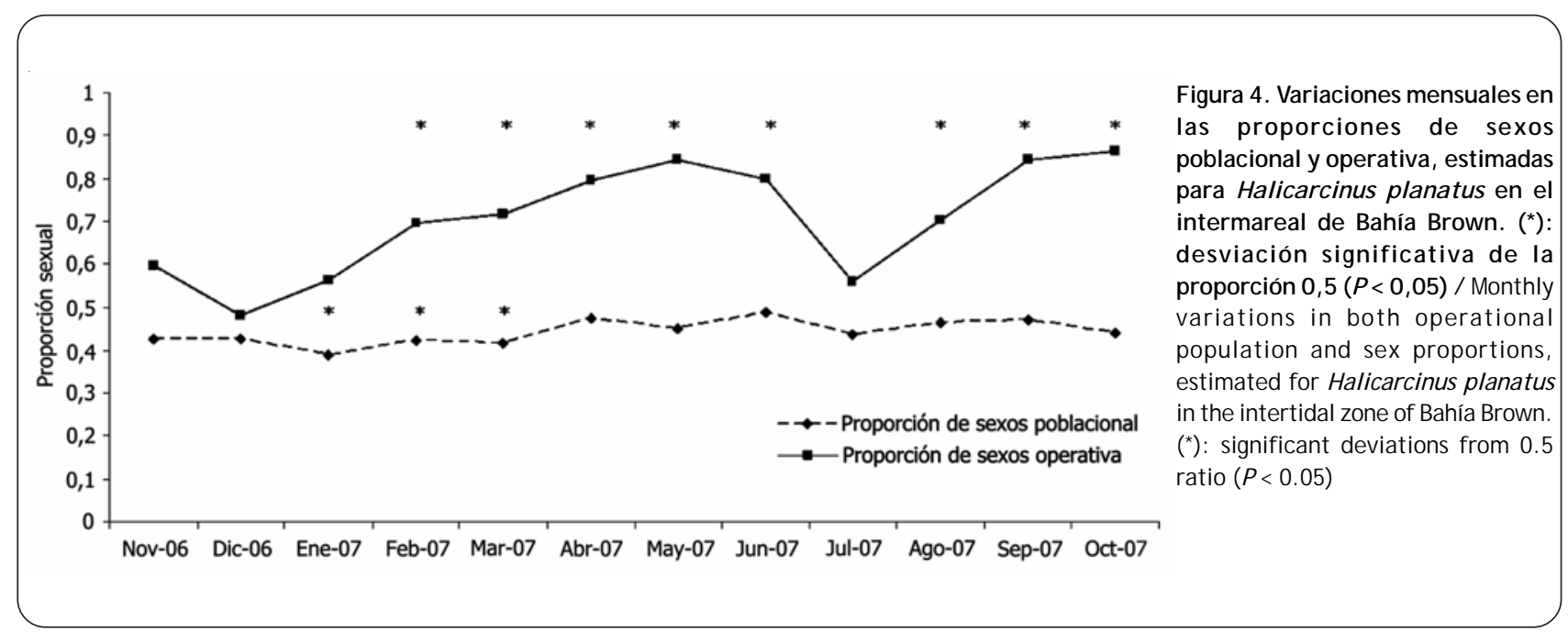


Tabla 2. Proporción de sexos en función del tamaño de Halicarcinus planatus en el intermareal de Bahía Brown. Se muestran los resultados del test $\chi^{2}$ para probar la proporción 0,5 y su probabilidad asociada / Sex proportion as a function of size in Halicarcinus planatus in the Bahía Brown intertidal zone. Results of the $\chi^{2}$ tests for 0.5 proportions and their associated probability are showed

\begin{tabular}{|c|c|c|c|c|c|}
\hline $\begin{array}{l}\text { Ancho de caparazón } \\
\qquad(\mathrm{mm})\end{array}$ & $\begin{array}{l}\text { Proporción } \\
\text { sexual }\end{array}$ & $\begin{array}{c}\mathrm{N}^{\circ} \\
\text { machos }\end{array}$ & $\begin{array}{c}\mathrm{N}^{\circ} \\
\text { hembras }\end{array}$ & $\chi^{2}$ & $P$ \\
\hline $1,50-1,99$ & 0,67 & 2 & 1 & 0,17 & 0,68 \\
\hline $2,00-2,49$ & 0,46 & 13 & 15 & 0,00 & 1,00 \\
\hline $2,50-2,99$ & 0,50 & 77 & 77 & 0,01 & 0,91 \\
\hline $3,00-3,49$ & 0,48 & 114 & 126 & 0,21 & 0,65 \\
\hline $3,50-3,99$ & 0,68 & 226 & 107 & 21,23 & $<0,001$ \\
\hline $4,00-4,49$ & 0,46 & 126 & 149 & 0,80 & 0,37 \\
\hline $4,50-4,99$ & 0,50 & 158 & 157 & 0,00 & 0,97 \\
\hline $5,00-5,49$ & 0,47 & 109 & 123 & 0,31 & 0,58 \\
\hline $5,50-5,99$ & 0,53 & 110 & 98 & 0,24 & 0,62 \\
\hline $6,00-6,49$ & 0,34 & 101 & 197 & 15,22 & $<0,001$ \\
\hline $6,50-6,99$ & 0,46 & 78 & 92 & 0,42 & 0,51 \\
\hline $7,00-7,49$ & 0,53 & 134 & 119 & 0,33 & 0,56 \\
\hline $7,50-7,99$ & 0,35 & 61 & 114 & 7,61 & $<0,05$ \\
\hline $8,00-8,49$ & 0,30 & 69 & 159 & 17,67 & $<0,001$ \\
\hline $8,50-8,99$ & 0,31 & 53 & 120 & 12,69 & $<0,001$ \\
\hline $9,00-9,49$ & 0,32 & 63 & 137 & 13,42 & $<0,001$ \\
\hline $9,50-9,99$ & 0,36 & 43 & 77 & 4,35 & $<0,05$ \\
\hline $10,00-10,49$ & 0,48 & 22 & 24 & 0,00 & 1,00 \\
\hline $10,50-10,99$ & 0,61 & 25 & 16 & 0,60 & 0,44 \\
\hline $11,00-11,49$ & 0,67 & 8 & 4 & 0,17 & 0,68 \\
\hline $11,50-11,99$ & 0,71 & 10 & 4 & 0,60 & 0,44 \\
\hline $12,00-12,49$ & 0,83 & 5 & 1 & 0,38 & 0,54 \\
\hline $12,50-12,99$ & 1 & 2 & 0 & 0,00 & 1,00 \\
\hline $13,00-13,49$ & 1 & 3 & 0 & 0,22 & 0,64 \\
\hline $13,50-13,99$ & 1 & 1 & 0 & 0,67 & 0,41 \\
\hline $14,00-14,49$ & & - & - & & \\
\hline $14,50-15,00$ & 1 & 1 & 0 & 0,67 & 0,41 \\
\hline
\end{tabular}

DistribuCión Y ESTRUCTURA POBLACIONAL EN OTROS Sitios Del CANAL BEAGLE

Halicarcinus planatus se presentó en la mayoría de los ambientes intermareales examinados en este estudio (Tabla 1). Sólo en dos sitios no se encontraron individuos, Baliza Lapataia y Ensenada Este (Fig. 1). A su vez, estuvieron presentes en los grampones de Macrocystis pyrifera y en las cuerdas de cultivo de mejillones.

La densidad de individuos determinada en el interior de Bahía Brown (sitio 5, Fig. 1) fue de 72,8 \pm 69,8 machos $\cdot \mathrm{m}^{-2}$ y 72,0 $\pm 58,1$ hembras $\cdot \mathrm{m}^{-2}$ con una proporción de sexos insesgada $\left(\chi^{2}=0,97 ; P=0,32\right)$. Por otra parte, se hallaron individuos indiferenciados sexualmente de tamaño pequeño, pertenecientes al estadio cangrejo I (1,65 $\pm 0,11 \mathrm{~mm}$ AC). Los tamaños mejor representados dentro de Bahía Brown fueron $<4 \mathrm{~mm}$ AC para ambos sexos, representando $72,8 \%$ y $82,4 \%$ del total de hembras y machos, respectivamente.

No se encontraron machos en los restantes intermareales muestreados, pero sí se hallaron en las cuerdas de cultivo de mejillones y en los grampones de Macrocystis pyrifera. Los machos estuvieron presentes en los grampones de $M$. pyrifera pero se registraron variaciones estacionales en la proporción de sexos. Sólo en invierno la proporción de sexos no se desvió de 0,5. Durante el resto del año, esta proporción estuvo sesgada hacia las hembras con ausencia de machos en otoño (Tabla 3). Por otra parte, ambos sexos estuvieron presentes en las cuerdas de mejillones en una proporción $0,5\left(\chi^{2}=0,54 ; P=0,46\right)$.

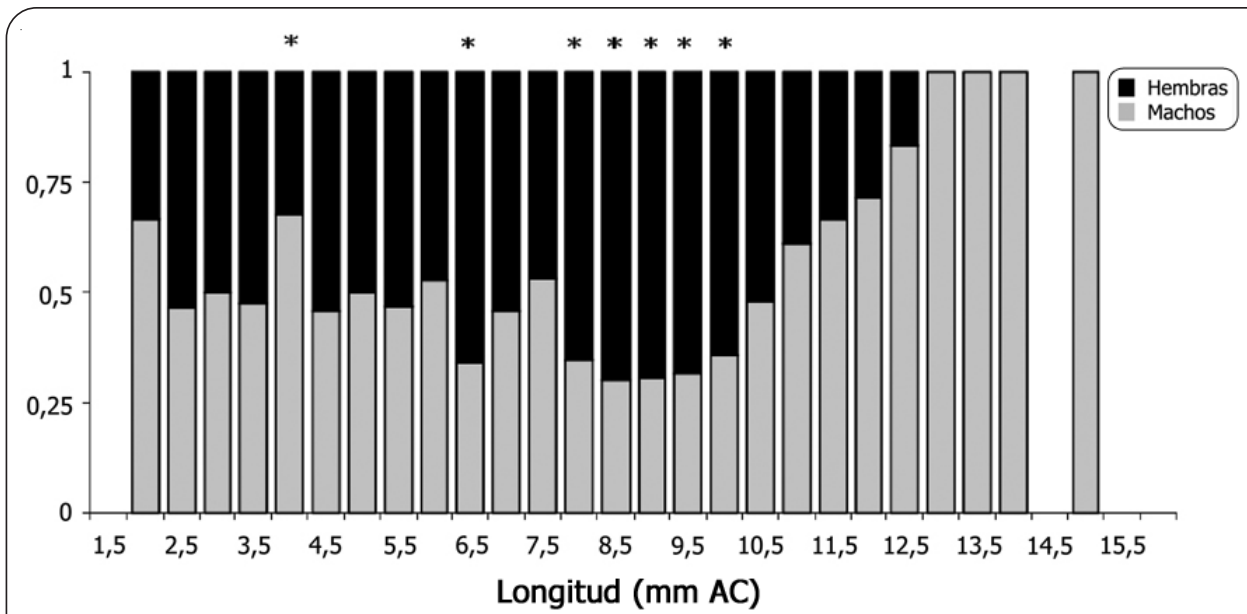

Figura 5. Proporción de sexos de acuerdo al tamaño de Halicarcinus planatus. $(*)$ : representa desvíos significativos de la proporción 0,5 . El tamaño muestral de cada clase de tamaño es la suma de machos y hembras de la Tabla 2. En las abscisas, los valores representan los límites superiores de los intervalos / Sex proportions according to size classes for Halicarcinus planatus. $(*)$ : represents significant deviations from 0.5 ratio. Sample size is the sum of males and females from Table 2. On the abscisas, values represent higher limits of intervals 


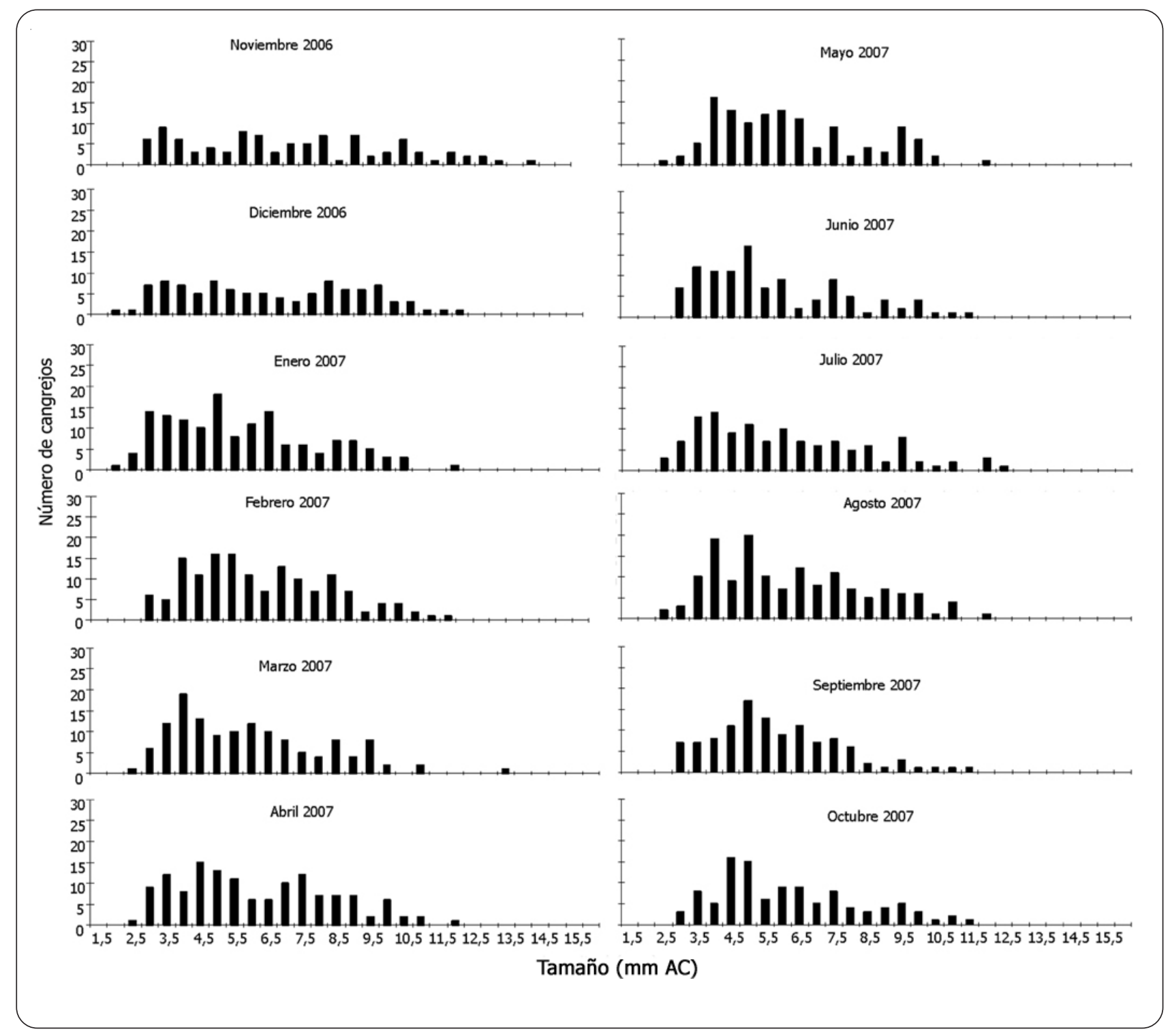

Figura 6. Distribuciones de frecuencias de tamaño de machos de Halicarcinus planatus en el intermareal de Bahía Brown entre noviembre 2006-octubre 2007 / Size frequency distributions for Halicarcinus planatus males in the intertidal zone of Bahía Brown between November 2006-October 2007

Tabla 3. Proporción de sexos de Halicarcinus planatus en distintos sitios y ambientes del Canal Beagle. Se muestran los resultados del test $\chi^{2}$ para probar la proporción 0,5 y su probabilidad asociada / Sex proportion of Halicarcinus planatus in different sites and environments of the Beagle Channel. Results of the $\chi^{2}$ tests for 0.5 proportions and their associated probability are showed

\begin{tabular}{llcrr}
\hline Sitio & Estación & Proporción de sexos & $\chi^{2}$ & $P$ \\
\hline Cuerdas Mytilus edulis & Invierno & 0,43 & 0,54 & 0,46 \\
Extremo Bahía Brown & Verano & 0,65 & 5,51 & $<\mathbf{0 , 0 5}$ \\
\multirow{2}{*}{ Grampones Macrocystis pyrifera } & Verano & 0,12 & 58,18 & $<\mathbf{0 , 0 0 1}$ \\
& Otoño & 0,00 & 8,82 & $<\mathbf{0 , 0 1}$ \\
& Invierno & 0,47 & 0,09 & 0,77 \\
& Primavera & 0,20 & 19,95 & $<\mathbf{0 , 0 0 1}$ \\
\hline
\end{tabular}




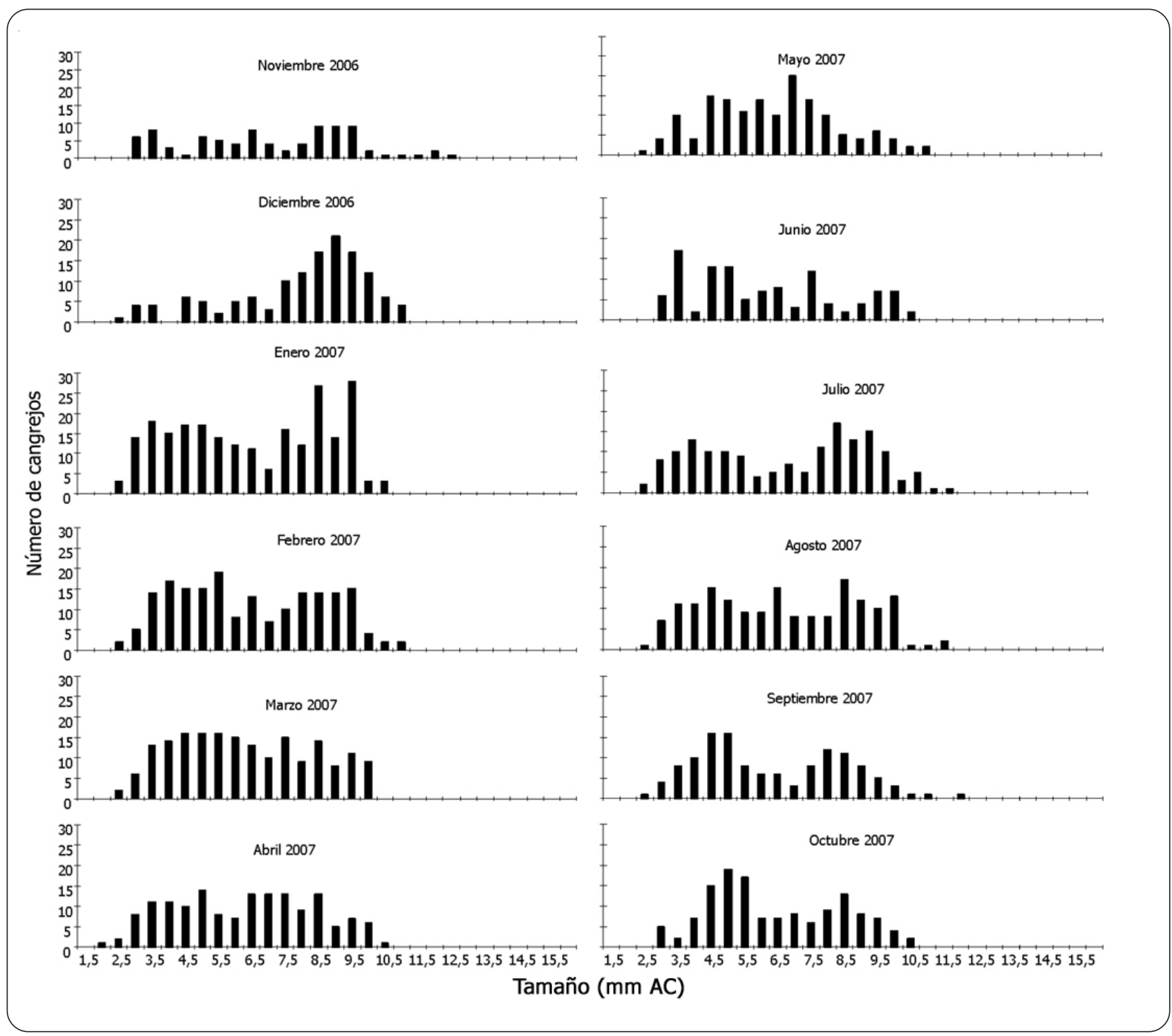

Figura 7. Distribuciones de frecuencias de tamaño de hembras de Halicarcinus planatus en el intermareal de Bahía Brown entre noviembre 2006-octubre 2007 / Size frequency distributions for Halicarcinus planatus females in the intertidal zone of Bahía Brown between November 2006-October 2007

En el intermareal de Ensenada Oeste (sitio 2 Fig. 1) todas las clases de tamaño de las hembras entre 7 y 10 mm ocurrieron en alta frecuencia. En Lapataia (sitio 1, Fig. 1), las clases con mayor proporción de individuos estuvieron entre los 8,5 y 10,5 mm (90\%). Al igual que en Lapataia, la mayor proporción de individuos en el intermareal de Baliza Davison se observó en las clases 8$10 \mathrm{~mm}$ (64\%) pero, a diferencia de Lapataia, estuvieron también representadas clases intermedias (3,5-6,5 mm) con $\sim 33 \%$ de los individuos (Fig. 8). Las frecuencias más altas en las cuerdas de cultivo de mejillones, fueron de $<5 \mathrm{~mm}$ para ambos sexos, aunque clases $\geq 7 \mathrm{~mm}$ estuvieron representadas sólo en las hembras.

\section{Discusión}

Existen registros sobre los cambios temporales en la abundancia y distribución de H. planatus (islas Kerguelen $49^{\circ} \mathrm{S}$, Richer de Forges 1977). Sin embargo, los datos sobre variaciones temporales mostrados en este estudio son los primeros para la población más austral en el rango de su distribución.

Los estudios poblacionales de himenosomátidos muestran abundancias altas para la mayoría de las especies de la familia (revisión en Lucas 1980) (Tabla 4). Sin embargo, la abundancia de Halicarcinus planatus en 


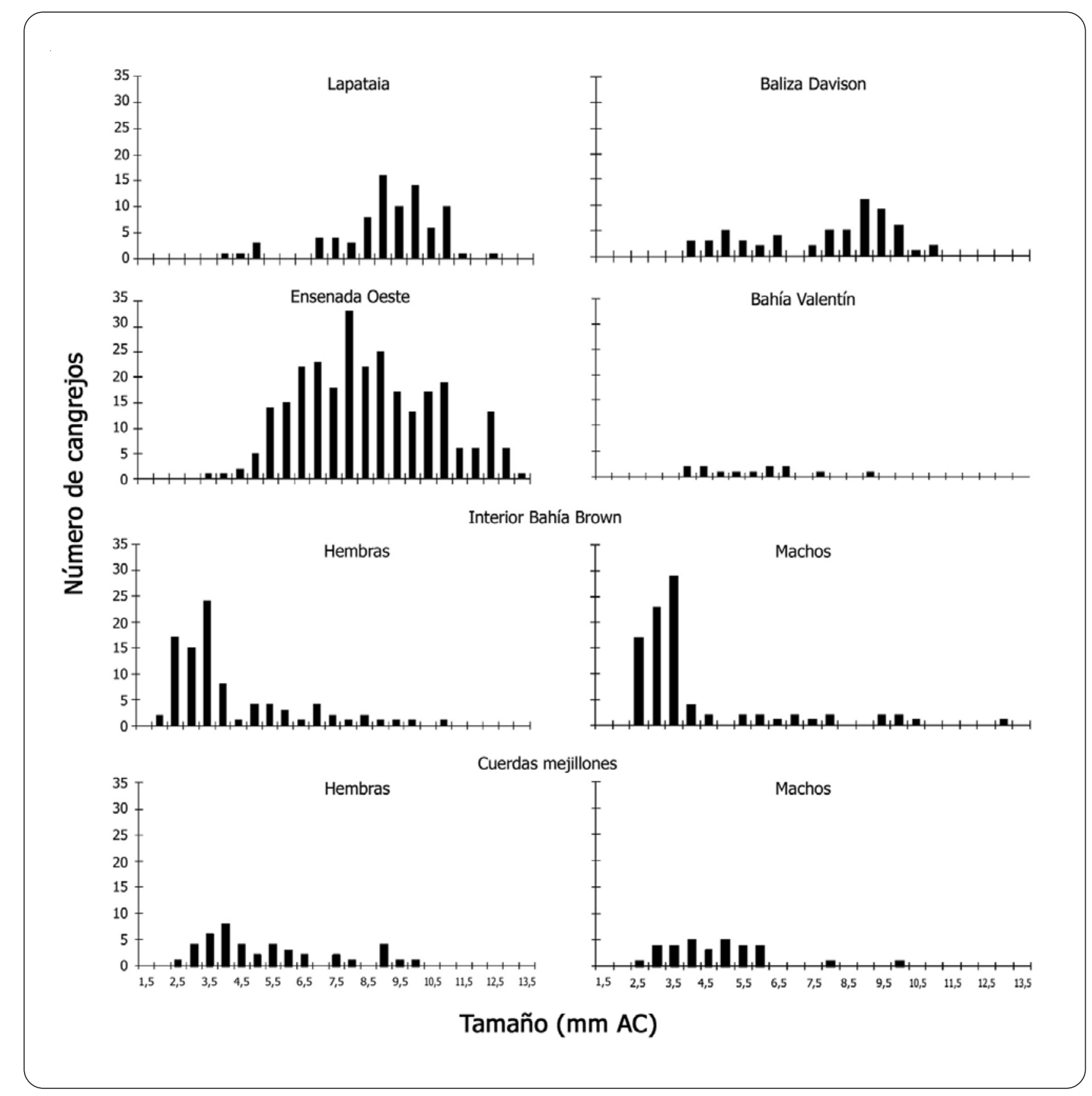

Figura 8. Distribuciones de frecuencias de tamaño de Halicarcinus planatus en diversos intermareales del Canal Beagle y en las cuerdas de cultivo de mejillones / Size frequency distributions of Halicarcinus planatus in different intertidals and mussel culture lines in the Beagle Channel 
Tabla 4. Densidad de diferentes especies de himenosomátidos en distintos ambientes / Density of hymenosomatid species from different environments

\begin{tabular}{cccc}
\hline Especie & Ind. $\mathrm{m}^{-2}$ & Sustrato & Fuente \\
\hline Halicarcinus cooki & $20-40$ & Corallina & Melrose (1968) \\
H. innominatus & $100-150$ & Bancos de mejillones & Melrose (1968) \\
H. innominatus & $30-40$ & Pozas de marea & Melrose (1968) \\
H. whitei & 8 & Arena & Melrose (1968) \\
H. varius & $40-60$ & Sargassum & Melrose (1968) \\
H. varius & 40 & Playas protegidas & Wood (1968) \\
H. varius & 120 & Zostera & Wood (1968) \\
H. planatus & $23-135$ & Bancos de mejillones & Richer de Forges (1977) \\
H. planatus & 226 & Intermareal rocoso & Este estudio \\
H. rostratus & $10-160$ & Heterozostera & Lucas (1980) \\
H. ovatus & $10-110$ & Heterozostera & Lucas (1980) \\
Hymenosoma orbiculare & 2 & Fondo lacustre & Boltt (1969) fide (Lucas 1980) \\
Neohymenicus pubsescens & $20-60$ & Arrecifes & Melrose (1968) \\
\hline
\end{tabular}

Bahía Brown resulta aún mayor a otros himenosomátidos con una densidad media anual de 225,8 \pm 94 ind. $\mathrm{m}^{-2}$. Estas altas densidades en el intermareal podrían estar relacionadas con dos factores, por un lado, con la baja mortalidad de estadios larvales avanzados (Zoea III; Lucas 1975), evidenciada en su abundancia en la comunidad zooplanctónica (Lovrich 1999) y el éxito en el reclutamiento; y por otro lado, con los procesos oceanográficos de Bahía Brown. La circulación de corrientes dentro de la bahía presenta rasgos particulares (Hernando et al. 2008¹), con características geográficas que limitarían el intercambio de agua con las adyacencias del canal, reduciendo la dispersión larval fuera de la bahía (Lucas 1975).

El patrón en la variación temporal de la abundancia fue coincidente con el observado en la población de Halicarcinus planatus de las islas Kerguelen (Richer de Forges 1977). Al igual que en estas islas, la abundancia total de la especie en el intermareal de Bahía Brown muestra sus valores más altos durante dos períodos: enero y agosto, y mínimos en junio. La estimación de la abundancia total para cada sexo, mostró que el principal incremento de abundancia en enero se relaciona exclusivamente con el aumento de la abundancia de las hembras inmaduras. Por su parte, las hembras ovígeras presentan sus mayores densidades en diciembre y julio, en correspondencia con una disminución de hembras inmaduras. Esta correspondencia indica que durante julio podría ocurrir la muda de pubertad terminal (Diez \&
Lovrich 2010) ya que la proporción de hembras maduras no disminuye al aumentar la de hembras ovígeras.

En el intermareal de Bahía Brown, durante los meses de verano, la proporción de sexos de Halicarcinus planatus estuvo sesgada significativamente hacia las hembras, aunque los machos siempre están presentes. La ausencia de variaciones en la densidad de machos podría indicar que no hay migraciones una vez arribados al intermareal, probablemente relacionado con la ausencia de Macrocystis pyrifera en Bahía Brown. Esto contrasta con lo que podría ocurrir en otras playas del Canal Beagle, como también en otras poblaciones patagónicas, donde la ausencia de machos resulta normal (e.g., Vinuesa \& Ferrari 2008a, b).

La proporción de sexos operativa (PSO) se desvió significativamente de 0,5 durante la mayor parte del año. Esta desviación indica que existe mayor número de machos que de hembras aptos para el apareamiento, planteando que puede existir una fuerte competencia intrasexual por las hembras disponibles (Kvarnemo \& Ahnesjö 2002). Los cangrejos braquiuros presentan básicamente tres modos de apareamiento diferentes: apareamiento exclusivamente con hembras blandas (postmuda), apareamiento exclusivamente con hembras duras (intermuda) o apareamientos con ambos tipos de hembras indistintamente (Hartnoll 1969). Las hembras de $H$. planatus se aparean en períodos de intermuda (Richer de Forges 1977). La alta proporción de machos observada durante casi todo el año es indicativa que existen machos 
listos para aparearse en ese período. En consecuencia, la cópula podría ocurrir casi todo el año porque, al igual que Halicarcinus cookii, los machos de $H$. planatus podrían aparearse incluso con hembras ovígeras en intermuda (van den Brink \& McLay 2009) debido a la capacidad de las hembras de almacenar esperma en sus espermatecas (Lucas 1980, van den Brink \& McLay 2009).

La fuerte competencia entre machos de Halicarcinus planatus por el apareamiento de las hembras receptivas indica que podría estar actuando la selección sexual en dos niveles. Por un lado, la quela de $H$. planatus es sexualmente dimórfica y más grande en machos que en hembras del mismo tamaño (Diez \& Lovrich 2010), y generalmente este dimorfismo sexual en el tamaño de la quela es asociado con la selección sexual (Orensanz \& Gallucci 1988). Además, un segundo cambio morfométrico se observa en las quelas de machos que alcanzan los tamaños más grandes (Diez \& Lovrich 2010) que podría conferirles mayor probabilidad de apareamiento (Juanes et al. 2008). Sin embargo, a pesar que los machos de menor tamaño y fisiológicamente maduros, son capaces de aparearse (obs. pers.), estarían en desventaja frente a los enfrentamientos con otros machos de quelas más grandes (van den Brink \& McLay 2009). Estos machos pequeños podrían tener una estrategia 'pure search' (Baeza \& Thiel 2007) al estar favorecidos por un incremento en su agilidad y en la tasa de encuentro con hembras receptivas. Por otro lado, la capacidad de las hembras de almacenar el esperma (i.e., hasta 15 cópulas distintas en su espermateca; Lucas 1980), indica que la selección sexual podría actuar a través de la competencia espermática (Birkhead et al. 2009). Es conocido que la probabilidad de competencia espermática aumenta con el incremento de la proporción de sexos operativa (Jirotkul 1999).

Cuando la proporción de sexos poblacional es analizada en función del tamaño, los desvíos significativos de la proporción 0,5 se sesgan hacia las hembras en las clases de tamaño intermedias (7,5 - $10 \mathrm{~mm}$ AC). Las hembras tienen su madurez morfométrica y fisiológica en este rango (Diez \& Lovrich 2010). Si se considera que esta especie presenta muda terminal (Richer de Forges 1977, Vinuesa \& Ferrari 2008a), las hembras se acumularían en estas clases de tamaño, originando la diferencia en la proporción de sexos. Asimismo, existen otros factores que contribuirían a la diferencia en la proporción de sexos en relación con el tamaño como las tasas de crecimiento y mortalidad diferencial entre sexos (Johnson 2003). A pesar de la disponibilidad de refugio en el intermareal de Bahía Brown, las altas densidades de Halicarcinus planatus promueven su vulnerabilidad hacia los predadores aéreos como la gaviota cocinera Larus dominicanus (Bertellotti et al. 2003) y los skúas Stercoraruis skua (Richer de Forges 1977). En consecuencia, la depredación también podría desviar la proporción de sexos si existieran diferencias morfológicas asociadas al comportamiento entre machos y hembras (Iribarne \& Martínez 1999, Johnson 2003).

Los resultados sobre las distribuciones de frecuencias de tamaño de Halicarcinus planatus en Bahía Brown sugieren que existirían dos períodos de reclutamiento o ingreso de juveniles desde su lugar de asentamiento, uno en verano (enero-febrero) y el otro a fines del otoñoprincipios del invierno (mayo-junio). Sin embargo, no se registraron individuos indiferenciados o juveniles tempranos (Cangrejo I) con la metodología utilizada. Podría ocurrir que una vez producida la metamorfosis los individuos se asienten en el ambiente submareal (i.e., mejillonar, ver en 'Distribución y estructura poblacional en otros sitios del Canal Beagle') y migren hacia el intermareal, luego de diferenciarse sexualmente a tamaños entre 2-2,8 mm AC (Richer de Forges 1977). Este proceso migratorio ontogenético ha sido documentado para varias especies de cangrejos, e.g., Carcinus maenas, Chionoecetes opilio, Chaceon affinis y Xantho poressa (Comeau et al. 1998, López-Abellán et al. 2002, Moksnes et al. 2003, Spivak et al. 2010). En este contexto, la ausencia de bosques de Macrocystis pyrifera en Bahía Brown, cuyos grampones sirven de refugio para muchas especies, incluída H. planatus (Adami \& Gordillo 1999, Ríos et al. 2007) explicaría que alcanzado cierto tamaño, los juveniles de $H$. planatus migren hacia el intermareal en búsqueda de refugio, y protegerse de sus predadores acuáticos, como los peces Harpagifer bispinis, Patagonotothen tessellata y Austrolycus depressiceps (Fernández et al. 2010²).

Halicarcinus planatus se encuentra en distintos ambientes del Canal Beagle y aunque en las playas del canal habitan otras especies de decápodos (e.g., Acanthocyclus albatrossis, 20 ind. $\mathrm{m}^{-2}$, Avalos 2005), los resultados sugieren que $H$. planatus es el cangrejo más

2Fernández D, L Riccialdelli, S Newsome, M Victorio \& E González. 2010. Estudio de la trama trófica de Bahía Lapataia ante la invasión del salmón chinook de los ríos Ovando y Lapataia (Parque Nacional Tierra del Fuego). Libro de resúmenes IV Reunión Binacional de Ecología, Buenos Aires. 
abundante en los intermareales del Canal Beagle. Sin embargo, dependiendo del ambiente, presenta variaciones en su abundancia y estructura poblacional, ya que presenta altas densidades en un mejillonar ubicado en la zona intermareal del extremo interior de Bahía Brown, pero está prácticamente ausente de otro mejillonar en Ensenada Este. Las altas frecuencias de individuos pequeños observados sugieren que en el interior de Bahía Brown se podría producir el asentamiento de juveniles y posteriormente una posible migración hacia otras zonas de la bahía. Los bancos de mejillones intermareales son hábitats favorables para el reclutamiento de varias especies de cangrejos intermareales (Navarrete et al. 2008). En la Bahía Brown, este proceso podría relacionarse con los cultivos de mejillones desarrollados en sus aguas, ya que los bancos de mejillones en la zona intermareal están presentes desde el inicio de la instalación de las balsas de cultivo, que podrían proveer de larvas a los sustratos arenosos intermareales (Lovrich obs. pers.). Posteriormente y al adquirir un mayor tamaño, los juveniles de $H$. planatus migrarían hacia el intermareal rocoso, con mayor disponibilidad de alimento y refugio.

Las características oceanográficas de la Bahía Brown, con intercambio de agua reducido con las adyacencias del Canal Beagle y los cultivos de mejillones originan que la zona sea muy favorable para la supervivencia con altas densidades de Halicarcinus planatus. Los mejillones y/ o sus valvas vacías parecen ser un sustrato adecuado para el asentamiento y reclutamiento de los juveniles. En las líneas de cultivo de mejillones de la bahía también se encuentran individuos de tamaños pequeños sugiriendo asentamiento también en ese ambiente. Esto no es algo novedoso para la especie ya que en los sistemas de cultivo de mitílidos del sur de Chile ( $\left.41^{\circ} 47^{\prime} \mathrm{S} 73^{\circ} 02^{\prime} \mathrm{O}\right)$ también se encuentran ejemplares de $H$. planatus pero con tamaños mayores que el de los individuos en sus hábitats naturales (López et al. 1987).

Excepto en los intermareales rocosos y el de mejillones de Bahía Brown, la ausencia de machos de Halicarcinus planatus es una característica común en el resto de los intermareales estudiados en el Canal Beagle. Sin embargo, los machos se encuentran en los grampones de los bosques de Macrocystis pyrifera, cuya presencia es común hasta los 40 m de profundidad (Kühnemann 1970). Estos grampones otorgarían a los machos el hábitat críptico que no encuentran en otras zonas intermareales. Este patrón también se observa en la población del estuario de la ría Deseado (Vinuesa \& Ferrari 2008a, b) y en las islas Kerguelen (Richer de Forges 1977) pero no se corresponden con la presencia de machos en la población del extremo norte de su distribución, Mar del Plata (Diez obs. pers.). Este tipo de segregación por sexo es común en algunas familias de cangrejos (e.g., Geryonidae) donde hembras y machos pueden ubicarse en ambos extremos de la distribución vertical de una especie (e.g., Chaceon affinis, López-Abellán et al. 2002).

Nuestros resultados evidencian que Halicarcinus planatus es uno de los crustáceos decápodos más abundantes en el Canal Beagle, con densidades locales incluso superiores a una de las especies clave del canal, Munida gregaria (promedio: 152 ind. $100 \mathrm{~m}^{-2}$, Tapella 2002). Las fluctuaciones de la densidad y las distribuciones de frecuencia de tamaño muestran el ingreso a la población de individuos de tamaños pequeños durante el verano y fines del otoño. Sin embargo, no se cuenta con evidencias directas de reclutamiento, debido a la ausencia de estadios Cangrejos I. Los juveniles se asentarían en un ambiente diferente del hábitat del adulto y migrarían al intermareal rocoso al alcanzar cierto tamaño. Por otra parte, la estructura poblacional de $H$. planatus denota una proporción de sexos operativa sesgada hacia las hembras, con ausencia total de machos en ciertos ambientes, hecho que sugiere una fuerte competencia entre machos de esta especie en el Canal Beagle.

\section{Agradecimientos}

Agradecemos a F. Tapella, MC Romero, MP Sotelano, P. Pérez Barros y G. Scioscia por la ayuda en el trabajo de campo. Este estudio fue financiado por la Agencia Nacional de Promoción Científica y Tecnológica (PICT 01-1385). E. Spivak proporcionó valiosas sugerencias para la mejora del manuscrito. MJD tiene una beca doctoral del CONICET, Argentina. Este estudio está basado en el trabajo de MJD como parte de lo requerimientos para el grado de Doctor en la Universidad Nacional de Mar del Plata, Argentina.

\section{LITERATURA CITADA}

Adami ML \& S Gordillo. 1999. Structure and dynamics of the biota associated with Macrocystis pyrifera (Phaeophyta) from Beagle Channel, Tierra del Fuego. Scientia Marina 63(Suppl. 1): 183-191.

Avalos MC. 2005. Estructura poblacional y morfometría de Acanthocyclus albatrossis Rathbun 1898 en Bahía Varela, Canal Beagle, Tierra del Fuego. Tesis de grado, Facultad de Ciencias Exactas y Naturales, Universidad Nacional de Mar del Plata, Mar del Plata, 62 pp. 
Baeza JA \& M Thiel. 2007. The mating system of symbiotic crustaceans. A conceptual model based on optimality and ecological constraints. En: Duffy JE \& M Thiel (eds). Evolutionary ecology of social and sexual systems. Crustaceans as model organisms, pp. 249-267. Oxford University Press, Oxford.

Bertellotti M, G Pagnoni \& P Yorio. 2003. Comportamiento de alimentación de la gaviota cocinera (Larus dominicanus) durante la temporada no reproductiva en playas arenosas de Península Valdés, Argentina. Hornero 18(1): 37-42.

Birkhead TR, DJ Hosken \& S Pitnick. 2009. Sperm biology. An evolutionary perspective, 683 pp. Academic Press, New York.

Comeau M, GY Conan, F Maynou, G Robichaud, JC Therriault \& M Starr. 1998. Growth, spatial distribution, and abundance of benthic stages of the snow crab (Chionoecetes opilio) in Bonne Bay, Newfoundland, Canada. Canadian Journal of Fisheries and Aquatic Science 55: 262279.

Chuang CTN \& PKL Ng. 1994. The ecology and biology of Southeast Asian false spider crabs (Crustacea: Decapoda: Brachyura: Hymenosomatidae). Hydrobiologia 285(1): 8592.

Diez MJ \& GA Lovrich. 2010. Reproductive biology of the crab Halicarcinus planatus (Brachyura, Hymenosomatidae) in sub-Antarctic waters. Polar Biology 33: 389-401.

Diez MJ, MC Romero, S Obenat, M Albano \& F Tapella. 2009. Distribution of benthic invertebrates in the Beagle Channel, Argentina. Anales del Instituto de la Patagonia 37(2): 29-40.

Hartnoll RG. 1969. Mating in the Brachyura. Crustaceana 16: 161-181.

Iribarne O \& M Martinez. 1999. Predation on the southwestern Atlantic fiddler crab (Uca uruguayensis) by migratory shorebirds (Pluvialis dominica, P. squatarola, Arenaria interpres and Numenius phaeopus). Estuaries and Coasts 22(1): 47-54.

Jirotkul M. 1999. Operational sex ratio influences female preference and male-male competition in guppies. Animal Behaviour 58(2): 287-294.

Johnson PTJ. 2003. Biased sex ratios in fiddler crabs (Brachyura, Ocypodidae): A review and evaluation of the influence of sampling method, size class, and sex-specific mortality. Crustaceana 76(5): 559-580.

Johnston K \& B Robson. 2005. Habitat use by the hymenosomatid crab Amarinus lacustris (Chilton) in two south-eastern Australian rivers. Marine and Freshwater Research 56: 37-44.
Juanes F, K Lee, A McKnight \& K Kellogg. 2008. Claw allometry in green crabs, Carcinus maenas: heterochely, handedness, and sex. Marine Biology 153(4): 523-528.

Kühnemann O. 1970. Algunas consideraciones sobre los bosques de Macrocystis pyrifera. Physis 29: 273-296.

Kvarnemo C \& I Ahnesjö. 2002. Operational sex ratios and mating competition. En: Hardy I (ed). Sex ratios concepts and research methods, pp. 366-383. Cambridge University Press, New York.

López DA, O Huaiquian \& ML González. 1987. Sobre las poblaciones de Halicarcinus planatus (Fabricius) (Decapoda: Hymenosomidae) en el sur de Chile. Biota, Chile 3: 79-84.

López-Abellán L, JE Balguerías \& V Fernández-Vergaz. 2002. Life history characteristics of the deep-sea crab Chaceon affinis population off Tenerife (Canary Islands). Fisheries Research 58(2): 231-239.

Lucas JS. 1975. The larval stages of some Australian species of Halicarcinus (Crustacea, Brachyura, Hymenosomatidae). III Dispersal. Bulletin of Marine Science 25(1): 94-100.

Lucas JS. 1980. Spider crabs of the family Hymenosomatidae (Crustacea; Brachyura) with particular reference to Australian species: systematics and biology. Records of the Australian Museum 33(4): 148-247.

Moksnes P, O Hedvall \& T Reinwald. 2003. Settlement behavior in shore crabs Carcinus maenas: why do postlarvae emigrate from nursery habitats? Marine Ecology Progress Series 250: 215-230.

Navarrete S, M Parragué \& E Wieters. 2008. Local and meso-scale patterns of recruitment and abundance of two intertidal crab species that compete for refuges. Marine Biology 155(2): 223-232.

Orensanz JM \& VF Gallucci. 1988. Comparative study of postlarval life-history schedules in four sympatric species of Cancer (Decapoda: Brachyura: Cancridae). Journal of Crustacean Biology 8(2): 187-220.

Pérez-Barros P, F Tapella, MC Romero, J Calcagno \& G Lovrich. 2004. Benthic decapod crustaceans associated with captures of Munida spp. (Decapoda: Anomura) in the Beagle Channel, Argentina. Scientia Marina 68(2): 237-246.

Rahayu DL \& P Ng. 2004. The Hymenosomatidae (Crustacea, Decapoda,Brachyura) of Timika (Irian Jaya, Indonesia). Zoosystema 26(1): 87-94.

Richer de Forges B. 1977. Etude du crabe des Iles Kerguelen: Halicarcinus planatus (Fabricius). Comite National Francais des Recherces Antarctiques 42: 71-133.

Ríos C, W Arntz, D Gerdes, E Mutschke \& A Montiel. 2007. Spatial and temporal variability of the benthic assemblages associated to the holdfasts of the kelp Macrocystis pyrifera in the Straits of Magellan, Chile. Polar Biology 31(1): 89-100. 
Sokal RR \& FJ Rohlf. 1995. Biometry, the principles and practice of statistics in biological research, 887 pp. W.H. Freeman and Co, New York

Spivak ED, E Arévalo, JA Cuesta \& JI González-Gordillo. 2010. Population structure and reproductive biology of the stone crab Xantho poressa (Crustacea: Decapoda: Xanthidae) in the Corrales de Rota (south-western Spain), a humanmodified intertidal fishing area. Journal of the Marine Biological Association of the United Kingdom 90(2): 323334.

Tapella F. 2002. Reproducción, crecimiento, distribución y abundancia de la langostilla Munida subrugosa (Anomura: Galatheidae) del Canal Beagle, Tierra del Fuego, Argentina. Tesis doctoral, Universidad Nacional de Córdoba, Córdoba, 158 pp.
Van den Brink AM \& CL McLay. 2009. Use of the sterile males technique to investigate sperm competition, storage and use in a Pill box crab, Halicarcinus cookii (Brachyura: Hymenosomatidae). Journal of Crustacean Biology 29(1): 62-69.

Vinuesa J \& L Ferrari. 2008a. Postlarval development of Halicarcinus planatus females (Crustacea: Decapoda: Hymenosomatidae) in the estuary of the Deseado River, Argentina. Scientia Marina 72(1): 127-132.

Vinuesa J \& L Ferrari. 2008b. Reproduction of Halicarcinus planatus (Crustacea, Decapoda, Hymenosomatidae) in the Deseado River estuary, southwestern Atlantic Ocean. Marine Biology 154(2): 345-351.

Wilson K \& I Hardy. 2002. Statistical analysis of sex ratios: an introduction. En: Hardy I (ed). Sex ratios: Concepts and research methods, pp. 48-93. Cambridge University Press, New York.

Recibido el 25 de enero de 2011 y aceptado el 23 de marzo de 2011 